\title{
Sensitivity of solar wind mass flux to coronal temperature ${ }^{\star}$
}

\author{
D. Stansby ${ }^{1}$, L. Berčič ${ }^{2,3}$, L. Matteini ${ }^{4}$, C. J. Owen ${ }^{1}$, R. J. French ${ }^{1}$, D. Baker ${ }^{1}$, and S. T. Badman ${ }^{5,6}$ \\ ${ }^{1}$ Mullard Space Science Laboratory, University College London, Holmbury St. Mary, Surrey RH5 6NT, UK \\ e-mail: d.stansby@ucl.ac.uk \\ ${ }^{2}$ LESIA, Observatoire de Paris, PSL Research University, CNRS, UPMC University Paris 6, University Paris-Diderot, Meudon, \\ France \\ ${ }^{3}$ Physics and Astronomy Department, University of Florence, Sesto Fiorentino, Italy \\ ${ }^{4}$ Department of Physics, Imperial College London, London, SW7 2AZ, UK \\ 5 Physics Department, University of California, Berkeley, CA 94720-7300, USA \\ ${ }^{6}$ Space Sciences Laboratory, University of California, Berkeley, CA 94720-7450, USA
}

Received 29 October 2020 / Accepted 7 December 2020

\begin{abstract}
Solar wind models predict that the mass flux carried away from the Sun in the solar wind should be extremely sensitive to the temperature in the corona, where the solar wind is accelerated. We perform a direct test of this prediction in coronal holes and active regions using a combination of in situ and remote sensing observations. For coronal holes, a $50 \%$ increase in temperature from 0.8 to $1.2 \mathrm{MK}$ is associated with a tripling of the coronal mass flux. This trend is maintained within active regions at temperatures over $2 \mathrm{MK}$, with a four-fold increase in temperature corresponding to a 200 -fold increase in coronal mass flux.
\end{abstract}

Key words. Sun: corona - Sun: heliosphere - solar wind - stars: winds, outflows

\section{Introduction}

The Sun continuously loses mass through the solar wind. Although the rate of this mass loss is small at $2 \times 10^{-14} M_{\odot} \mathrm{yr}^{-1}$ (Cohen 2011), it plays an important role in transporting angular momentum away from the Sun, controlling the rate at which it spins down (Weber \& Davis 1967; Li 1999).

The solar wind mass flux can be predicted with simple hydrodynamic models, where the number density is supplied as a lower boundary condition in the corona and an equation of state relating the temperature and density is assumed (Parker 1958, 1960). For a given base number density and under spherical expansion, the mass flux depends on the temperature profile inside the sonic point via

$n_{\odot} v_{\odot}=n_{\odot} c_{\odot}\left(\frac{r_{\mathrm{c}}}{r_{\odot}}\right)^{2} \frac{c_{\odot}}{c_{\mathrm{c}}} \exp \left[-\frac{1}{2} \int_{r_{\odot}}^{r_{\mathrm{c}}} r_{\mathrm{c}} \frac{w^{2}}{c^{2}(r) r^{2}} \mathrm{~d} r\right]$,

where $c^{2} \propto T$ is the thermal speed, $w$ is the solar escape velocity, $r$ is radial distance from the centre of the Sun, $n$ is number density, c subscripts are values evaluated at the critical sonic point (where $v_{\mathrm{c}}=c_{\mathrm{c}}$ ), and $\odot$ subscripts are values evaluated at the solar surface (Parker 1964, Eq. (25)). An increase in $T$ results in a decrease of the integral, which in turn results in the increase in the mass flux. The exponential dependence of mass flux on tem-

\footnotetext{
${ }^{\star}$ Code to reproduce the figures presented in this Letter is available at https://github.com/dstansby/publication-code. PSP and WIND data are available from https://spdf.gsfc .nasa.gov/pub/ data, GONG data from https://gong2.nso.edu/oQR/zqs/, SDO and SOHO data from http://jsoc.stanford.edu/, and EIS data from http://solarb.mssl.ucl.ac.uk/SolarB/.
}

perature means that, under spherical expansion, small variations in coronal temperature should result in large variations in mass flux. However, such large variations are not seen in the solar wind mass flux at 1 AU (Leer et al. 1982; Withbroe 1989; Goldstein et al. 1996).

The resolution of this apparent inconsistency involves two competing effects that cancel each other out: Areas with strong magnetic fields in the corona undergo stronger heating that drives increased mass fluxes, but stronger magnetic fields also undergo more super-radial expansion, resulting in a diluting of the mass over a larger area (Wang 2010). Correcting for radial magnetic field expansion and calculating near-Sun coronal (as opposed to solar wind) mass fluxes can be done routinely using magnetic field models, and it has been shown that the mass flux in the corona spans many orders of magnitude (Wang 1995, 2010; Schwadron \& McComas 2008). Correlating these large variations with temperature changes is challenging, however, as coronal temperatures are hard to reliably measure remotely (e.g. Habbal et al. 1993; Esser et al. 1995) and in situ measurements of solar wind temperatures at 1 AU have been significantly distorted from their coronal values (e.g. Marsch et al. 1983; Stansby et al. 2019b; Maksimovic et al. 2020).

In this Letter, we perform such a direct comparison using spectroscopic observations of two active regions and a newly proposed in situ proxy for coronal temperature in three coronal hole streams (Berčič et al. 2020). Section 2 briefly discusses the methods used to calculate coronal mass fluxes, and Sects. 3.1 and 3.2 present the solar wind streams and temperature measurements for coronal holes and active regions, respectively. Section 4 presents the main results, showing that a four-fold increase in coronal temperature is associated with a 200 -fold 
Table 1. Summary of the parameters and data sources of the five solar wind streams.

\begin{tabular}{ccccccc}
\hline \hline Stream & $|\boldsymbol{B}|_{\odot}(\mathrm{G})$ & $|\boldsymbol{B}|_{\mathrm{sw}} r^{2} / r_{\odot}^{2}(G)$ & $n_{\mathrm{sw}} v_{\mathrm{sw}} r^{2}\left(10^{35} \mathrm{sr}^{-1} \mathrm{~s}^{-1}\right)$ & $n_{\odot} v_{\odot} r_{\odot}^{2}\left(10^{35} \mathrm{sr}^{-1} \mathrm{~s}^{-1}\right)$ & $T_{\text {corona }}(\mathrm{MK})$ & Solar wind, $B_{\odot}, T_{\text {corona }}$ data \\
\hline S1 & 1.54 & 1.10 & 0.66 & 0.92 & 1.13 & PSP, HMI, PSP \\
S2 & 1.54 & 0.99 & 0.40 & 0.63 & 0.93 & PSP, HMI, PSP \\
S3 & 1.61 & 1.61 & 0.38 & 0.38 & 0.79 & PSP, HMI, PSP \\
AR1 & 19.2 & 2.91 & 0.42 & 2.66 & 1.86 & WIND, MDI, EIS \\
AR2 & 255 & 1.47 & 0.43 & 69.2 & 2.28 & WIND, MDI, EIS \\
\hline
\end{tabular}

Notes. The median value is given where a range of values is measured within each stream.

increase in coronal mass flux. The results are discussed and put into the context of other studies in Sect. 5, with conclusions provided in Sect. 6.

\section{Methods}

In order to compare mass fluxes over a wide range of coronal temperatures, data from both coronal holes and active regions were used. To infer coronal mass fluxes, in situ measurements of the solar wind mass flux were scaled back to their coronal values using the frozen-in theorem (e.g. Wang 2010):

$n_{\odot} v_{\odot}=n_{\mathrm{sw}} v_{\mathrm{sw}} \frac{\left|\boldsymbol{B}_{\odot}\right|}{\left|\boldsymbol{B}_{\mathrm{sw}}\right|}$,

where $n$ is the number density, $v$ is the radial velocity, and $\boldsymbol{B}$ is the magnetic field. An $s w$ subscript denotes a quantity measured in the solar wind and an $\odot$ subscript denotes a quantity evaluated at the base of the corona.

Different methods were used to measure the coronal temperatures of the coronal holes and active regions. For coronal holes, it is hard to reliably determine coronal temperatures with remote sensing data (e.g. Habbal et al. 1993; Wendeln \& Landi 2018), so a new method that provides a local in situ proxy for the coronal temperature was used. For active regions, hotter temperatures (and therefore higher ultraviolet emission intensities) allowed the use of remote sensing spectroscopy to estimate the coronal temperature.

\section{Data}

In this section, the choice of discrete solar wind streams is discussed, along with the data used to estimate coronal mass fluxes and temperatures for each source type. A summary of the data collected for each stream and the various data sources are given in Table 1.

\subsection{Coronal holes}

\subsubsection{Choice of streams}

Data taken by Parker Solar Probe (PSP, Fox et al. 2016) during its first perihelion were used to compare the properties of three coronal hole streams. One-minute averaged magnetic field data were taken from the FIELDS instrument suite (Bale et al. 2016) level 2 data. Solar wind proton core density and velocity data were taken from the Solar Wind Electron Alpha and Proton (SWEAP) suite (Kasper et al. 2016) level 3 data, with one-minute mean values taken to align the plasma data with the magnetic field data.

Magnetic mapping during the first perihelion pass shows PSP was initially connected to a small equatorial coronal hole and
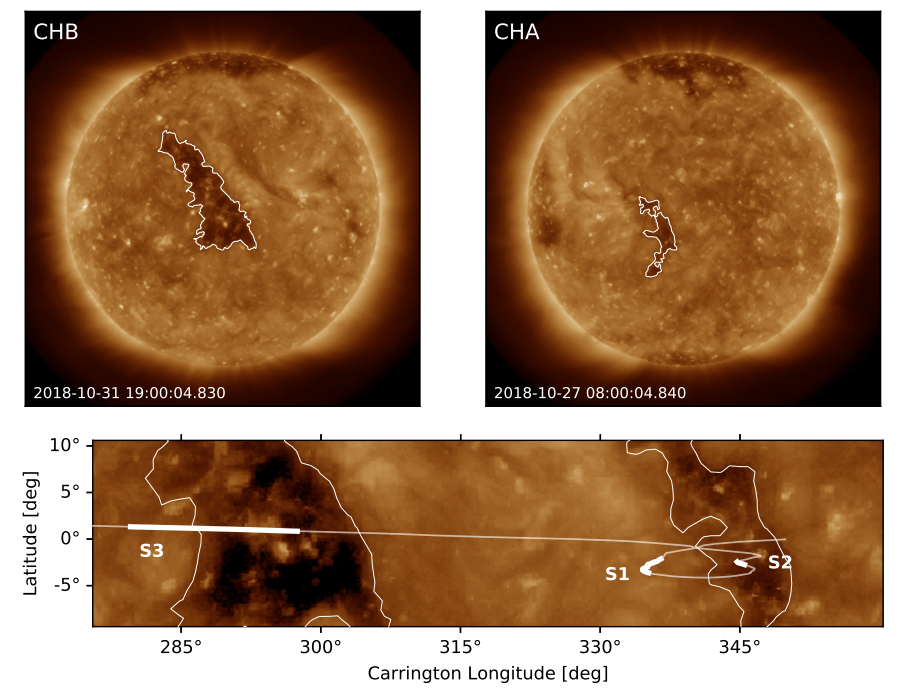

Fig. 1. Overview of coronal holes along the orbit of PSP during perihelion 1. Top panel: two coronal holes sampled by PSP, with contours showing the identified coronal hole with an intensity threshold at $50 \mathrm{DN} \mathrm{s}^{-1}$. The $y$-axis is aligned with solar north in both images, and the colour scale is clipped at $3000 \mathrm{DN} \mathrm{s}^{-1}$. Bottom panel: Carrington map of the same two coronal holes, with the white line showing the trajectory of PSP ballistically backmapped to $2.5 r_{\odot}$. Labelled areas of the trajectory are the in situ data intervals selected for analysis.

was subsequently connected to a second larger equatorial coronal hole (Badman et al. 2020). These are respectively labelled "CHA" and "CHB", and Fig. 1 shows images of them taken by the Atmospheric Imaging Assembly (AIA, Lemen et al. 2012) on board the Solar Dynamics Observatory (SDO, Pesnell et al. 2012). The top-left panel shows the large coronal hole, and the top-right panel shows the smaller coronal hole. Both were isolated using an intensity contour at $50 \mathrm{DN} \mathrm{s}^{-1}$.

The bottom panel of Fig. 1 shows a synoptic map with the trajectory of PSP ballistically backmapped to $2.5 R_{\odot}$. In this co-rotating Carrington coordinate system, the spacecraft moved from right to left with time, performing a loop at the closest approach. The highlighted portions of the trajectory indicate the three intervals selected for further analysis, labelled $\mathrm{S}\{1,2,3\}$. The first two were located on either side of the perihelion loop over the small coronal hole, and the third interval was located over the large coronal hole.

Figure 2 shows an overview of solar wind parameters measured by PSP, with the three streams indicated with coloured bands. The top panel shows the solar wind speed. The streams marked in Figs. 1 and 2 were selected to have a relatively constant velocity, avoiding any stream interaction regions (e.g. Perrone et al. 2018). Stream S1 was the lowest velocity interval during perihelion. Although it had very slow 

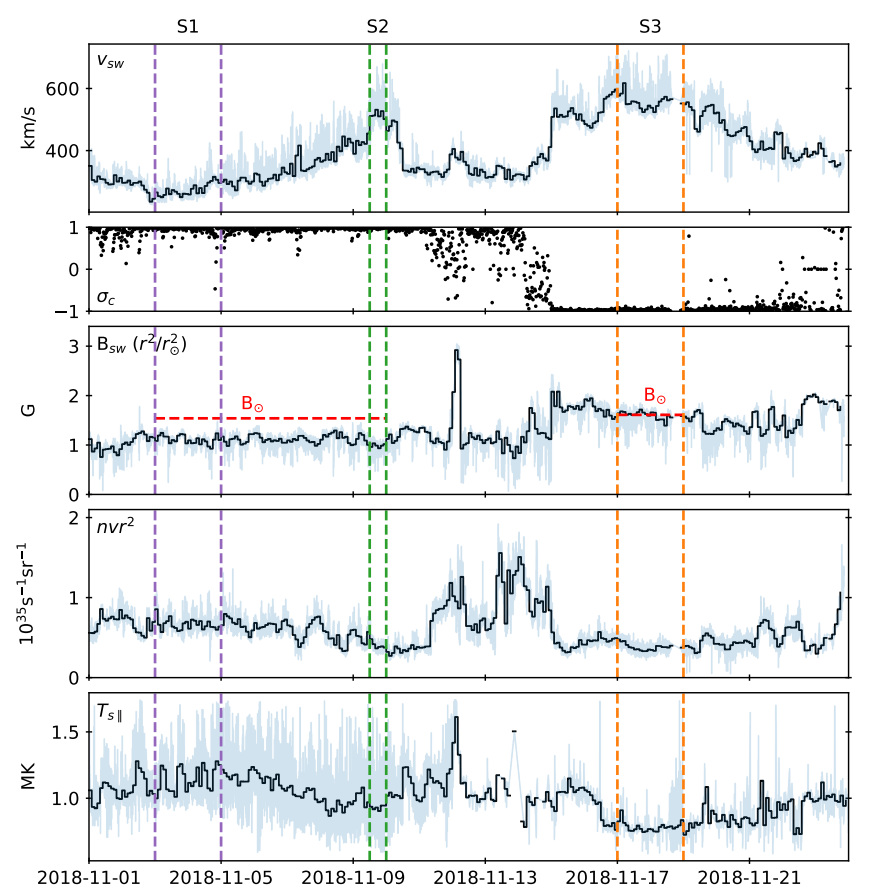

Fig. 2. PSP measurements during perihelion 1, with vertical dashed lines bounding the three distinct streams discussed in the text. Light grey lines are one-minute measurements and the solid black lines are two-hour averages. Top panel: solar wind velocity, and second panel: cross helicity calculated on a 20-min scale. Third panel: in situ magnetic field, scaled by $\left(r / r_{\odot}\right)^{2}$ to make it directly comparable to coronal magnetic field strengths. Horizontal dashed lines show the corresponding magnetic field strength in the source coronal holes. Fourth panel: solar wind mass flux, and fifth panel: parallel electron strahl temperature.

speeds $\left(\approx 250 \mathrm{~km} \mathrm{~s}^{-1}\right), \mathrm{S} 1$ was strongly Alfvénic, as shown in the second panel, which plots cross helicity (calculated as in Stansby et al. 2019a, on a 20-min timescale). This supports the idea that $\mathrm{S} 1$ originated in a coronal hole (e.g. D'Amicis et al. 2018; Stansby et al. 2020b). It originated away from the centre CHA (see Fig. 1), consistent with a large expansion factor and therefore a slower speed. Stream S2 had a higher speed at around $500 \mathrm{~km} \mathrm{~s}^{-1}$. This stream was also highly Alfvénic and originated in the same coronal hole as S1. This part of the trajectory was directly over the centre of the small coronal hole, likely consistent with a smaller expansion factor and therefore a faster speed. Stream S3 was another high speed stream at the end of the interval. This was a typical fast solar wind stream, highly Alfvénic and with speeds around $500 \mathrm{~km} \mathrm{~s}^{-1}$. It originated from the large coronal hole (CHB) shown in the top-left panel of Fig. 1 and was measured by PSP during the outbound leg of its orbit, at $0.3-0.5 \mathrm{AU}$.

\subsubsection{Coronal magnetic field}

The magnetic field strength in each coronal hole was calculated from photospheric magnetograms measured by the Heliospheric Magnetic Imager (HMI, Scherrer et al. 2012) on the SDO. Coronal hole boundaries were taken from intensity thresholds at $50 \mathrm{DN} \mathrm{s}^{-1}$ on AIA $193 \AA$ Amages (shown in Fig. 1), and the mean magnetic field (corrected for projection effects) within these boundaries was calculated using the method from Hofmeister et al. (2017). Taking a single average value assumes a spatially isotropic magnetic field strength with each coronal hole, which is a good assumption at the base of the corona where the plasma beta is $\ll 1$ (Peter et al. 2006).
The third panel of Fig. 2 shows coronal magnetic field strengths in context with the in situ data. Dashed red lines show the coronal hole magnetic field strengths, and the solar wind data are scaled by $\left(r / r_{\odot}\right)^{2}$. This allows the expansion factor to be visualised, defined as $f=\left(B_{\odot} r_{\odot}^{2}\right) /\left(B_{\mathrm{sw}} r^{2}\right)$. For streams S1 and S2, the photospheric magnetic field was larger than the scaled in situ field, giving an expansion factor $>1$. In contrast, stream S3 had almost identical photospheric and scaled in situ field strengths, giving an expansion factor of unity.

\subsubsection{Coronal temperature}

The fourth panel of Fig. 2 shows the parallel strahl electron temperature (Berčič et al. 2020). This is defined as the gradient of high energy electrons (the strahl) in velocity space. Under adiabatic expansion, and due to the low collisionality of the high energy electrons, the strahl temperature should be conserved from when the corona was last collisionally dominated to where it is measured the solar wind, giving a proxy for the coronal temperature (Berčič et al. 2020). Although there is a large scatter between individual measurements, there are clear trends visible across the whole interval. During S1, the temperature started relatively high and then gradually declined as the solar wind speed increased until S2. During S3, the measurements were sparser, but on average this interval had lower temperatures than the previous intervals.

\subsection{Active regions}

\subsubsection{Choice of streams}

Two active regions were analysed, both of which had been previously studied, remotely and in situ by van Driel-Gesztelyi et al. (2012) and Stansby et al. (2020a), respectively. These studies used magnetic modelling and ballistic backmapping to identify the in situ solar wind intervals at $1 \mathrm{AU}$, corresponding to each active region. In situ data were measured by the Solar Wind Experiment (SWE, Ogilvie et al. 1995) and the Magnetic Field Investigation (MFI, Lepping et al. 1995) on board WIND, from 2008 January 12 14:00 UT to 2008 January 13 12:00 UT for AR1 and 2013 January 24 00:00 UT to 2013 January 25 00:00 UT for AR2.

\subsubsection{Coronal magnetic field}

To isolate the areas in the corona responsible for feeding the solar wind, open-closed field maps were calculated around each active region by tracing field lines through a potential field source surface (PFSS, Altschuler \& Newkirk 1969; Schatten et al. 1969) model. The models were calculated from Global Oscillation Network Group (GONG, Harvey et al. 1996; Plowman \& Berger 2020) synoptic photospheric magnetic field maps using the pfsspy software package (Stansby et al. 2020c), with a source surface radius at $2.5 R_{\odot}$.

The open-closed field contour for each active region is shown over-plotted in Fig. 3. To measure the coronal magnetic field, the open field regions were isolated on high resolution line of sight field maps from the Michelson Doppler Imager (MDI, Scherrer et al. 1995) for AR1 and from the HMI for AR2. The average photospheric field within the open field contour was calculated as in Sect. 3.1.2.

\subsubsection{Coronal temperature}

Spatially resolved spectrographic data from Hinode/EUV Imaging Spectrometer (EIS Kosugi et al. 2007; Culhane et al. 2007) 

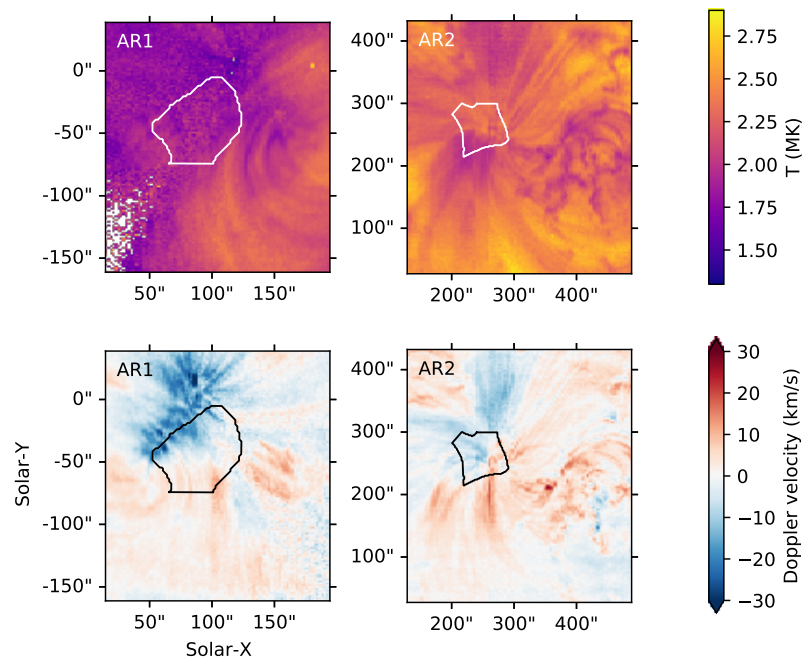

Fig. 3. Observations of the two active regions used in this study. Top panels: Doppler velocity, with negative (blue) values indicating flows away from the Sun. Bottom panels: plasma temperature. The black and white contours outline regions of open magnetic fields. We note that the spatial scales are different for the two regions, with the area of the open field region 4 times larger in AR2 than in AR1.

were used to measure electron temperatures in the active regions. The EIS data were prepped and fitted using the SolarSoftWare eis_prep and eis_auto_fit routines. The Fe XIII 202.04 to Fe XII 195.11 $\AA$ lines observed by EIS are a temperaturesensitive line pair, with good sensitivity at active region temperatures (Del Zanna \& Mason 2018, Sect. 11.1). Using the theoretical ratio of these lines computed in CHIANTI v8 (Del Zanna et al. 2015), temperature maps were calculated for the two active regions. These electron temperature maps are shown in the top two panels of Fig. 3. As an additional check on whether coronal material was flowing into the solar wind (e.g. Harra et al. 2008; Marsch et al. 2008), line of sight Doppler velocity maps were also calculated for the Fe XIII $202.04 \AA$ line $^{1}$, shown in the bottom panels of Fig. 3. The distributions of temperatures within each active region were taken from pixels within the open field contour, which had negative Doppler velocities (i.e. material was flowing away from the Sun).

\section{Results}

Using Eq. (2), point by point measurements of solar wind mass flux divided by magnetic field strength were multiplied by the average photospheric source magnetic field to give a distribution of coronal mass fluxes for each source. The distribution of coronal temperatures was taken either from the strahl parallel temperatures within coronal holes (see Sect. 3.1.3) or the active region temperatures determined through spectroscopy (see Sect. 3.2.3).

Figure 4 shows the variation of coronal mass flux with coronal temperature across the five different streams analysed here. Within the coronal hole streams, the fastest stream had the lowest temperatures $(\approx 0.8 \mathrm{MK})$ and the lowest mass fluxes $(\approx 0.3 \times$ $\left.10^{35} \mathrm{~s}^{-2} \mathrm{sr}^{-1}\right)$. In contrast, the slow wind interval had temperatures around $50 \%$ higher than this, and mass fluxes around $200 \%$ higher. The intermediate coronal hole interval confirms this trend, lying between the other intervals in both temperature and mass flux. The active regions both had higher temperatures,

\footnotetext{
1 With the reference wavelength (i.e. zero velocity point) set assuming zero average shift over the entire map.
}

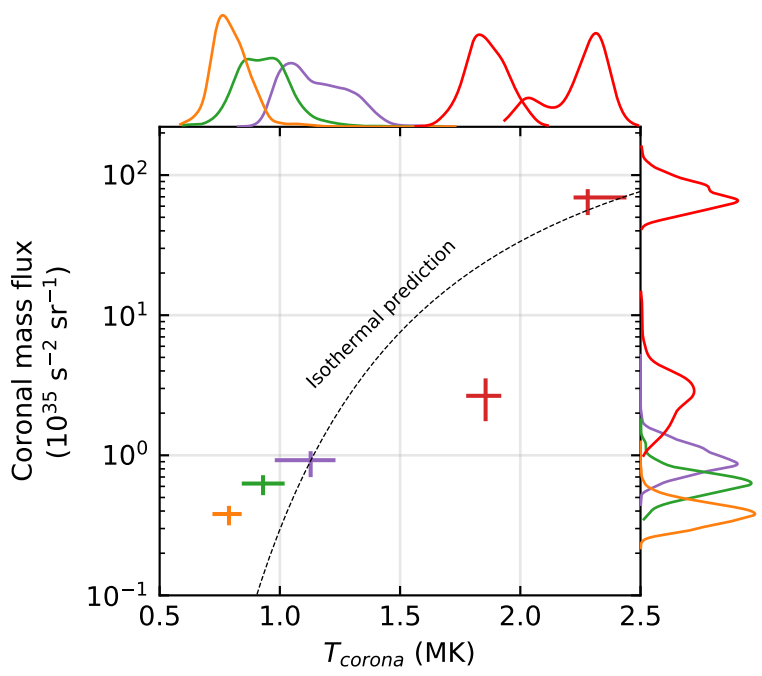

Fig. 4. Joint distributions of coronal temperature and coronal mass flux for the three coronal holes (lower left) and two active regions (upper right). Crosses are centred on the median values and span the 20th to 80th percentiles. Marginal 1D distributions show Gaussian kernel density estimations. The dashed line is an isothermal prediction for $|\boldsymbol{B}| \propto 1 / r^{2}$.

at 2 and $2.4 \mathrm{MK}$, and significantly higher mass fluxes, at 2 and $80 \times 10^{35} \mathrm{~s}^{-2} \mathrm{sr}^{-1}$, respectively. Between the coolest coronal hole and the hottest active region, an increase in temperature by a factor of around four results in an increase in the coronal mass flux by a factor of over $200^{2}$.

Making a quantitative comparison to theory is challenging since even in simple fluid models the evolution of magnetic field strength and temperature as a function of height must be provided as inputs. The simplest prediction comes from a fluid model that assumes both $B \propto 1 / r^{2}$ and an isothermal temperature profile, which reduces Eq. (1) to (see Parker 1964, Eq. (31)):

$n_{\odot} v_{\odot}=n_{\odot} c R_{c}^{2} \mathrm{e}^{-2\left(R_{\mathrm{c}}-1\right)}$,

where $R_{\mathrm{c}}=r_{\mathrm{c}} / r_{\odot}=w^{2} /\left(4 c^{2}\right) \approx(5.8 \mathrm{MK}) / T$ is the sonic point normalised to the solar radius. Taking a typical observed value of $n_{\odot}=2 \times 10^{8} \mathrm{~cm}^{-3}$ (Del Zanna \& Bromage 1999), this prediction is shown as the dashed line in Fig. 4. This model agrees qualitatively with the data, in the sense that it predicts the correct order of magnitude and large variations in the mass flux. However, the trend fails to accurately predict mass fluxes for the cool coronal holes and intermediate-temperature active region. This is unsurprising as both the magnetic field strength and temperature profiles are not constant in the corona; more accurate model assumptions need to be considered to understand if fluid models successfully predict mass loss rates.

\section{Discussion}

The observation that coronal mass flux is extremely sensitive to corona temperature agrees qualitatively with fluid theories of the solar wind, which also predict the correct order of magnitude for the mass flux. To make more accurate quantitative comparisons, observed magnetic field strengths and coronal temperature profiles need to be measured. In the corona, $|\boldsymbol{B}|$ can only be directly measured below about $1.5 r_{\odot}$, and even then measurement is limited to brighter areas away from coronal holes

\footnotetext{
2 We stress that this is the mass flux at the base of the corona; the solar wind mass flux does not vary by such large orders of magnitude.
} 
(Wiegelmann et al. 2014; Yang et al. 2020). This could be circumvented by density and velocity measurements (Bemporad 2017) or magnetic field orientation measurements (Boe et al. 2020), which can indirectly infer expansion factors in the corona Temperature profiles can be estimated using off-limb spectroscopy (e.g. Landi 2008; Cranmer 2020) or, again, using density observations to indirectly infer temperatures (Lemaire \& Stegen 2016).

In contrast to this study of individual solar wind streams, changes in mass flux and coronal temperatures can be measured over multiple 11-yr solar cycles. In the minimum between cycles 23 and 24, the mass flux in polar coronal holes was lower than the minimum between cycles 22 and 23 (McComas et al. 2008, 2013; Zerbo \& Richardson 2015). This reduction was accompanied by a reduction in oxygen charge state ratios, which implies a corresponding reduction in the coronal temperature (Zhao \& Fisk 2011; Schwadron et al. 2014). Our study agrees well with, and provides a stream by stream verification of, these long duration statistical variations.

The mass flux carried away from a star controls stellar spin down, with the angular momentum loss rate directly proportional to the mass loss rate (Weber \& Davis 1967). Indeed, the reduction in coronal temperatures and therefore solar wind mass flux between cycles 23 and 24 drove a similar reduction in the solar angular momentum loss (Finley et al. 2019a,b). Our results suggest that if there were a way to remotely measure the coronal temperature of the parts of stars in which stellar winds originate, it would be possible to predict the mass loss rate. Unfortunately, only globally integrated observations are available for other stars, which are dominated by closed-loop emission (Cohen 2011; Mishra et al. 2019). However, our observations can be used to place new constraints on the mass fluxes predicted by solar and stellar wind models (e.g. Johnstone et al. 2015; Usmanov et al. 2018; Shoda et al. 2020).

\section{Conclusions}

We have presented a comparison of solar coronal temperatures and mass fluxes across three coronal holes and two active regions. A factor of four increase in coronal temperature results in a more than two orders of magnitude increase in mass flux in the solar wind sources studied, confirming that solar wind mass flux in the corona is extremely sensitive to the plasma temperature. This study provides a new insight into understanding solar mass loss via the solar wind, which in the future can be extended to large statistical studies and detailed theoretical comparisons.

Acknowledgements. D.S., C.J.O., and D.B. are supported by STFC grant ST/S000240/1. R.F. is supported by STFC grant ST/S50578X/1. Hinode is a Japanese mission developed and launched by ISAS/JAXA, collaborating with NAOJ as a domestic partner, NASA and UKSA as international partners. Scientific operation of the Hinode mission is conducted by the Hinode science team organised at ISAS/JAXA. This team mainly consists of scientists from institutes in the partner countries. Support for the post-launch operation is provided by JAXA and NAOJ (Japan), UKSA (U.K.), NASA, ESA, and NSC (Norway). We acknowledge the NASA Parker Solar Probe Mission and the FIELDS and SWEAP teams for use of data. We thank the anonymous referee for providing comments that helped improve presentation and interpretation of our results. Data processing was carried out with the help of astropy (Astropy Collaboration 2018), and sunpy (SunPy Community 2020). Figures were produced using Matplotlib (Hunter 2007).

\section{References}

Altschuler, M. D., \& Newkirk, G. 1969, Sol. Phys., 9, 131

Astropy Collaboration (Price-Whelan, A. M., et al.) 2018, AJ, 156, 123

Badman, S. T., Bale, S. D., Oliveros, J. C. M., et al. 2020, ApJS, 246, 23

Bale, S. D., Goetz, K., Harvey, P. R., et al. 2016, Space Sci. Rev., 204, 49
Bemporad, A. 2017, ApJ, 846, 86

Berčič, L., Larson, D., Whittlesey, P., et al. 2020, ApJ, 892, 88

Boe, B., Habbal, S., \& Druckmüller, M. 2020, ApJ, 895, 123

Cohen, O. 2011, MNRAS, 417, 2592

Cranmer, S. R. 2020, ApJ, 900, 105

Culhane, J. L., Harra, L. K., James, A. M., et al. 2007, Sol. Phys., 243, 19

D'Amicis, R., Matteini, L., \& Bruno, R. 2018, MNRAS, 483, 4665

Del Zanna, G., \& Bromage, B. J. I. 1999, J. Geophys. Res. Space Phys., 104, 9753

Del Zanna, G., \& Mason, H. E. 2018, Liv. Rev. Sol. Phys., 15, 5

Del Zanna, G., Dere, K. P., Young, P. R., Landi, E., \& Mason, H. E. 2015, A\&A, 582, A56

Esser, R., Brickhouse, N. S., Habbal, S. R., Altrock, R. C., \& Hudson, H. S. 1995, J. Geophys. Res., 100, 19829

Finley, A. J., Deshmukh, S., Matt, S. P., Owens, M., \& Wu, C.-J. 2019a, ApJ, 883,67

Finley, A. J., Hewitt, A. L., Matt, S. P., et al. 2019b, ApJ, 885, L30

Fox, N. J., Velli, M. C., Bale, S. D., et al. 2016, Space Sci. Rev., 204, 7

Goldstein, B., Neugebauer, M., Phillips, J., et al. 1996, A\&A, 316, 296

Habbal, S. R., Esser, R., \& Arndt, M. B. 1993, ApJ, 413, 435

Harra, L. K., Sakao, T., Mandrini, C. H., et al. 2008, ApJ, 676, L147

Harvey, J. W., Hill, F., Hubbard, R. P., et al. 1996, Science, 272, 1284

Hofmeister, S. J., Veronig, A., Reiss, M. A., et al. 2017, ApJ, 835, 268

Hunter, J. D. 2007, Comput. Sci. Eng., 9, 90

Johnstone, C. P., Güdel, M., Lüftinger, T., Toth, G., \& Brott, I. 2015, A\&A, 577, A27

Kasper, J. C., Abiad, R., Austin, G., et al. 2016, Space Sci. Rev., 204, 131

Kosugi, T., Matsuzaki, K., Sakao, T., et al. 2007, Sol. Phys., 243, 3

Landi, E. 2008, ApJ, 685, 1270

Leer, E., Holzer, T. E., \& Fla, T. 1982, Space Sci. Rev., 33, 161

Lemaire, J. F., \& Stegen, K. 2016, Sol. Phys., 291, 3659

Lemen, J. R., Title, A. M., Akin, D. J., et al. 2012, Sol. Phys., 275, 17

Lepping, R. P., Acũna, M. H., Burlaga, L. F., et al. 1995, Space Sci. Rev., 71, 207

Li, J. 1999, MNRAS, 302, 203

Maksimovic, M., Bale, S. D., Berčič, L., et al. 2020, ApJS, 246, 62

Marsch, E., Mühlhäuser, K. H., Rosenbauer, H., \& Schwenn, R. 1983, J. Geophys. Res., 88, 2982

Marsch, E., Tian, H., Sun, J., Curdt, W., \& Wiegelmann, T. 2008, ApJ, 685, 1262 McComas, D. J., Ebert, R. W., Elliott, H. A., et al. 2008, Geophys. Res. Lett., 35, 18

McComas, D. J., Angold, N., Elliott, H. A., et al. 2013, ApJ, 779, 2

Mishra, W., Srivastava, N., Wang, Y., et al. 2019, MNRAS, 486, 4671

Ogilvie, K. W., Chornay, D. J., Fritzenreiter, R. J., et al. 1995, Space Sci. Rev., 71,55

Parker, E. N. 1958, ApJ, 128, 664

Parker, E. N. 1960, ApJ, 132, 821

Parker, E. N. 1964, ApJ, 139, 72

Perrone, D., Stansby, D. Horbury, T. \& Matteini, L. 2018, MNRA, 483, 3730

Pesnell, W. D., Thompson, B. J., \& Chamberlin, P. C. 2012, Sol. Phys., 275, 3

Peter, H., Gudiksen, B. V., \& Nordlund, A. 2006, ApJ, 638, 1086

Plowman, J., \& Berger, T. 2020, Sol. Phys., 295, 143

Schatten, K. H., Wilcox, J. M., \& Ness, N. F. 1969, Sol. Phys., 6, 442

Scherrer, P. H., Bogart, R. S., Bush, R. I., et al. 1995, Sol. Phys., 162, 129

Scherrer, P. H., Schou, J., Bush, R. I., et al. 2012, Sol. Phys., 275, 207

Schwadron, N. A., \& McComas, D. J. 2008, ApJ, 686, L33

Schwadron, N. A., Goelzer, M. L., Smith, C. W., et al. 2014, J. Geophys. Res. Space Phys., 119, 1486

Shoda, M., Suzuki, T. K., Matt, S. P., et al. 2020, ApJ, 896, 123

Stansby, D., Horbury, T. S., \& Matteini, L. 2019a, MNRAS, 482, 1706

Stansby, D., Perrone, D., Matteini, L., Horbury, T. S., \& Salem, C. S. 2019b, A\&A, 623, L2

Stansby, D., Baker, D., Brooks, D. H., \& Owen, C. J. 2020a, A\&A, 640, A28

Stansby, D., Matteini, L., Horbury, T. S., et al. 2020b, MNRAS, 492, 39

Stansby, D., Yeates, A., \& Badman, S. T. 2020c, J. Open Source Softw., 5, 2732 SunPy Community (Barnes, a. W. T., et al.) 2020, ApJ, 890, 68

Usmanov, A. V., Matthaeus, W. H., Goldstein, M. L., \& Chhiber, R. 2018, ApJ, 865,25

van Driel-Gesztelyi, L., Culhane, J. L., Baker, D., et al. 2012, Sol. Phys., 281, 237

Wang, Y.-M. 1995, ApJ, 449, L157

Wang, Y.-M. 2010, ApJ, 715, L121

Weber, E. J., \& Davis, Leverett, J. 1967, ApJ, 148, 217

Wendeln, C., \& Landi, E. 2018, ApJ, 856, 28

Wiegelmann, T., Thalmann, J. K., \& Solanki, S. K. 2014, A\&ARv, 22, 78

Withbroe, G. L. 1989, ApJ, 337, L49

Yang, Z., Bethge, C., Tian, H., et al. 2020, Science, 369, 694

Zerbo, J.-L., \& Richardson, J. D. 2015, J. Geophys. Res. Space Phys., 120, 250

Zhao, L., \& Fisk, L. 2011, Sol. Phys., 274, 379 